\title{
The Mandibular Anterior Repositioning Appliance (MARA) - A report of three cases
}

SADJ November 2019, Vol. 74 No. 10 p561 - p570

JH Weber', P Botha², SH Dawjee ${ }^{3}$

\section{SUMMARY}

\section{Introduction}

The MARA (Mandibular Anterior Repositioning Appliance) is a fixed functional appliance used in the treatment of mandibular deficiencies.

\section{Aims and objectives}

To demonstrate the clinical capabilities, treatment effects and the expected duration of treatment when using the MARA, therefore creating awareness of the MARA as a treatment alternative to other functional appliances designed for correction of Class II malocclusions.

\section{Methods}

A retrospective study exploring the anteroposterior dimensional changes in the maxilla and mandible brought about by the MARA and the associated treatment time. The samples were the first three cases treated by a clinician inexperienced with the clinical application of the MARA and served as an ideal introduction to the treatment technique.

\section{Results}

In this study mandibular growth stimulation and temporomandibular joint remodeling may have been the main contributing factors in the resolution/improvement of the Class II malocclusions under treatment.

\section{Conclusion}

The MARA is a useful non-compliance appliance that produces exceptional treatment results when applied in combination with full fixed appliances. The changes

\section{Author affiliations:}

1. Johann H Weber: BChD (University of Pretoria), Private practice, Intercare Medical \& Dental Centre, Lynnwood, Pretoria, South Africa.

ORCID Number: 0000-0001-7157-0500

2. Piet Botha: $B C h D, M C h D$ (University of Pretoria), Consultant, University of Pretoria, Gauteng. Private practice, Xcelpark, Pretoria, South Africa.

ORCID Number: 0000-0002-6761-0466

3. Salahuddien M Dawjee: BChD, MDent (Orthodontics) (Medunsa), Head/Chief Specialist Department of Orthodontics, University of Pretoria, Pretoria, South Africa.

ORCID Number: 0000-0001-9245-6563

Corresponding author: Johann $\mathrm{H}$ Weber

Private practice, Intercare Medical \& Dental Centre, Lynnwood, Pretoria

South Africa.

Email: jhweber84@gmail.com, heinrich.weber@intercare.co.za

Author contributions:

1. Johann $\mathrm{H}$ Weber: Principal researcher, writing - $65 \%$

2. Piet Botha: Treatment, research and writing $-35 \%$

3. Salahuddien M Dawjee: Writing and advisor - 10\% observed were predominantly of a skeletal nature in the anteroposterior dimension. Maxillary growth restriction may also have played a role in the correction of these treated Class II abnormalities.

\section{Keywords}

MARA, Mandibular changes, Maxillary changes, SNA, SNB, ANB, WITS, Y-axis, Tweed angle, Facial angle, Mandibular growth.

\section{INTRODUCTION}

The idea of using a fixed functional appliance to stimulate mandibular growth was proposed by Angle and others of his peers many years ago, but the materials to make the concept an actuality in clinical practice were lacking. The development of stronger adhesives overcame this limitatation. The MARA was developed by Dr. D Toll and modified in 1994 by Dr. James E Eckhart to function as a fixed non-compliance appliance to correct Class II malocclusions. ${ }^{1,2}$

The decision as to which is the most effective technique to use in the treatment of growing patients with skeletal and dental Class II malocclusions has long been the source of considerable debate in the orthodontic literature. ${ }^{3}$ A scientific orthodontic study yielding insightful and meaningful results must ensure that the individual/samples studied must be of the same clinical and functional characteristics, age and gender.

Some widely utilized treatment techniques in the correction of Class II malocclusions include:

- space creation by performing selective extractions of teeth, especially upper first premolars, in order to camouflage the Class II malocclusion, or a reduction in upper tooth size by enamel stripping, by palatal expansion, utilization of the leeway space, or by orthopedic manipulations of the mandible and maxilla produced by headgear.

- functional appliance therapy together with full fixed appliances.

- temporary anchorage devices (TAD's) used in the distalization of molars.

- orthognathic surgery. ${ }^{4}$

The lack of success with removable functional appliance treatment has been attributed to a lack of patient compliance and the inability to control the amount and direction of mandibular growth. ${ }^{3}$ 
The MARA is a fixed functional appliance that eliminates the compliance factor. It has the added advantage of no inter-jaw restrictions, thereby allowing the patient to open his/her mouth unhindered in order to function normally. This device can be used in combination with full fixed appliances while skeletal correction is being achieved.

Although the MARA has been accepted as an effective non-compliance solution, it is not widely used as a functional non-extraction method of treatment of Class II malocclusions for various reasons. In one study, it was reported that the MARA was used clinically by only $5,8 \%$ of the orthodontists comprising the sample. ${ }^{5}$

The possibility of extra cost could play a role as well as operator and/or patient considerations with regards to handling/placement difficulties and the perceived longer chair time.

The aim of this study is to illustrate the MARA's clinical proficiencies, treatment effects and advantages by presenting three Class II cases, all selected and treated by the same operator.

The relatively uncomplicated nature of these cases was considered to make them an excellent introduction to the MARA as a supplementary treatment technique. The information gathered from the treatments may support the increased utilization of the MARA in the clinical orthodontic environment.

\section{Description of the MARA}

The MARA is a unique noncompliance Class II corrector that does not directly connect the maxillary and mandibular arches. It advances the mandible so that the patient functions in a new protrusive position.

It is claimed that this forced protrusive mandibular position stimulates bone remodeling and, if the patient is treated during the pubertal growth spurt, may result in the correction of the Class II malocclusion.

The MARA basically has six immoveable parts, including the transpalatal arch, that are manufactured and assembled as two separate units, fixed to the maxillary and mandibular first permanent molars respectively.

Stainless steel crowns are placed on the maxillary and mandibular first molar teeth. Ideally, a palatal bar can join the maxillary crowns, but it is not mandatory. In the mandible the crowns are joined by a rigid lingual arch that can be supported by bonded occlusal rests on the first premolars should clinical circumstances necessitate this.

The maxillary first molar stainless steel crown supports a large .062" $\times .062 "$ square tube on its buccal aspect, into which slides a .060"x.060" removable square elbow, the position of which can be varied anteroposteriorly by the placement of spacers which are available in 1, 2, 3 and $4 \mathrm{~mm}$ lengths. The vertical arm of the elbow extends occlusally about $10 \mathrm{cms}$ from the first maxillary molar and is then bent distally (Figure 1).
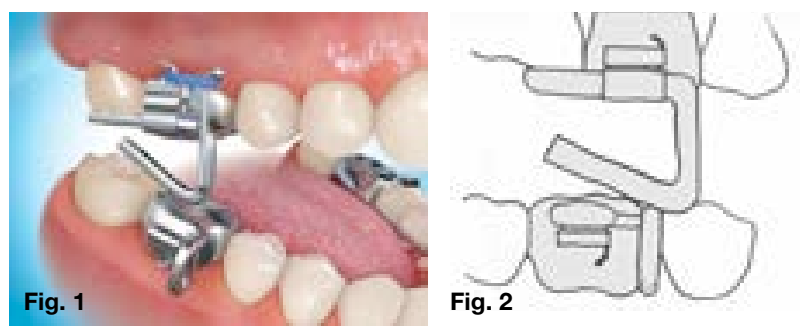

Figure 1. Components of the MARA.

Image Source: Components of the MARA, Tsibel G (http://www.getyourbraces.com/) Accessed on 27.08.14

Figure 2. MARA trying to occlude in Class II. Image Source: MARA in situ. 2007, Julie' D (http://www.ortho-concept.com/mara) Accessed on 27.08.14
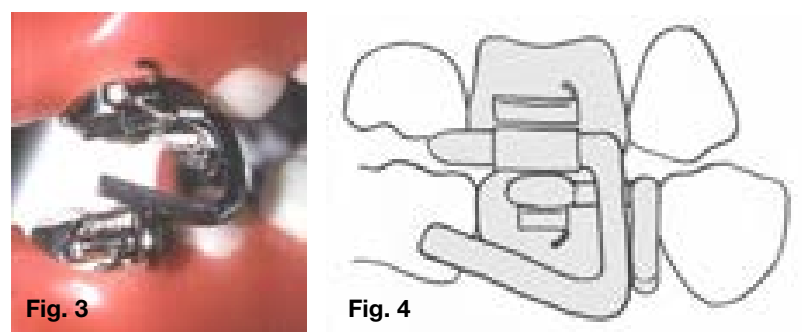

Figure 3. Trying to occlude in Class II. Image Source: Courtesy J. Eckhart.

Figure 4. Closed into Class I.

Image Source: Courtesy J. Eckhart.

To the mandibular first molar stainless steel crown is fixed a recurved rectangular wire arm that extends buccally about $5 \mathrm{mms}$ from the mesial aspect. This could be called the lower arm or shoulder 6 (Figure 1).

During attempted jaw closure into the habitual Class ॥ relationship, the inferior horizontal leg of the maxillary elbow contacts the mandibular arm, preventing functional occlusion in a retrusive position (Figures 2 and 3).

The Class II patient is therefore obliged to protrude the mandible until the lower arm is anterior to the vertical leg of the maxillary elbow component of the MARA, when closure into a forced protrusive position Class I position is possible (Figure 4).

The inferior horizontal leg of the upper elbow projects distally and prevents the patient from occluding into a Class II (Figure 3). Thus the TMJ is placed under constant strain similar to that caused by for example the Herbst appliance. ${ }^{6}$

\section{Advantages of the MARA}

The MARA has several advantages over the other noncompliance Class II appliances. These include:

- uncomplicated design - no inter-maxillary and mandibular connections. Sturdy and break resistant;

- aesthetically pleasing - no extra-oral headgear (decreased visibility);

- simple hygienic maintenance resulting in less oral mucosal infection/irritation;

- mandibular mobility is maintained - less functional movement impairment;

- fewer anchorage points resulting in less side-effects. ${ }^{7}$ 


\section{Side-effects and possible solutions}

The MARA is not without drawbacks. The treatment effects of the MARA in post-pubertal patients are limited due to lessened available mandibular growth. ${ }^{8}$

Side effects may involve:

\section{Mobility of the mandibular first molars}

Mobility of the lower first molars is caused when the upper elbows of the MARA contact the lower posterior aspect of the mandibular arms as the patient functions in the newly advanced position of the mandible. ${ }^{8}$ The solution to this side-effect is to simply place full fixed appliances and/or MARA occlusal rests on the mandibular first premolars during treatment to ensure stability. ${ }^{8}$

\section{Increased space between the mandibular anterior teeth}

Spacing of the mandibular anterior teeth may occur in patients treated with a MARA incorporating a lingual arch. The mandibular first molars may move forward (due to the maxillary elbows pushing on the back surface of the mandibular arms), the lingual arch will contact the posterior surfaces of the mandibular anterior teeth, causing anterior tipping and a flaring.

The solution to this complication is including occlusal rests which are bonded to the occlusal surfaces of the premolars and second molars thereby resisting mesial forces. Full bonding and selection of the correct negative torque (-6 degrees) for the lower incisors will provide more resistance to labial tipping. ${ }^{8}$

\section{Distal tipping and intrusion of the maxillary}

first molars together with possible extrusion or distal tipping of the maxillary second molars as both molars tip distally around their centroids (Figure 5).

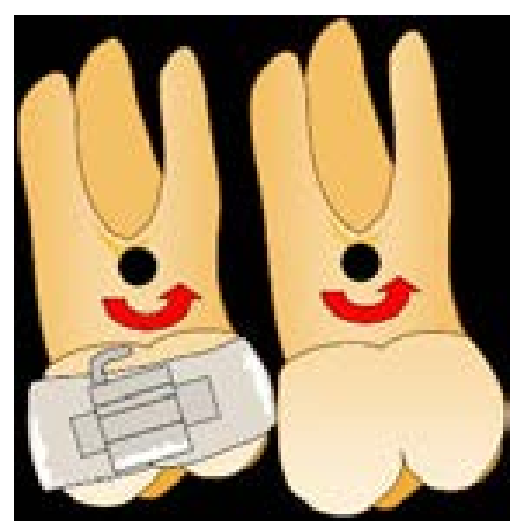

Figure 5. Distal tipping of molars.

When the MARA results in mandibular protrusion, the maxillary molars receive a distal force. This could cause distal tipping and intrusion of the maxillary first molar teeth. A simple solution would be to add a rest onto the occlusal surface of the maxillary second molar, extending distally from the first molar crown.

This will prevent extrusion of the second maxillary molars and intrusion as well as distal tipping of the maxillary first molars. ${ }^{8}$ Another aid to prevent distal tipping of the maxillary first molar is a transpalatal arch.
Class II relapse may also be a point of concern. It has been suggested that an adult patient treated with a MARA would have greater tendency to relapse than a child who has undergone the same treatment, as the correction in adults is mostly of a dento-alveolar nature.

Adults have larger muscle forces than children and this may result in a greater possibility for relapse if the apparent correction was partially due to temporary forward posturing of the mandible. If relapse is suspected, overcorrecting of the Class II malocclusion may be successful. ${ }^{8}$

In order to avoid distal tipping or dento-alveolar movement of the upper molar, the arch wire of the fixed orthodontic appliance should be cinched back behind the first molar. In cases where dento-alveolar movement is desired, as is needed in some adult cases, this is not done.

\section{MATERIALS AND METHODS}

This is a retrospective study of cephalometric and panoramic radiographs, study models and photographs of three adolescent male patients that presented with Class II malocclusions and were treated with the MARA.

The study aimed to determine the antero-posterior dentoskeletal treatment effects which were achieved and the respective durations of treatment. The cases selected were the first three such cases treated by the operator. They were deemed to be a suitable introduction to the use and clinical capabilities of the MARA.

All three cases were adolescent Caucasoid males with an average age of 16.7 years at the commencement of treatment. According to Proffit et al., the adolescent growth spurt in boys usually occurs between years 11-16 with the related physical changes peaking at around 14 years of age. ${ }^{4}$

Although the three patients were nearing the end of their adolescent growth spurt, it was determined that enough growth was still to be completed to warrant the use of the MARA.

The subjects received full arch fixed appliances concurrent with the MARA. One of the subjects presented a Class II division 2 in whom a lingual fixed appliance was placed in the maxilla.

Total treatment time (Table 2) referring to the duration of active treatment from the placement to removal dates of the full fixed appliances was as follows: patient 1 with a total treatment time of \pm 37 months (1109 days).

Treatment time for patient 2 from beginning to end was \pm 20 months (603 days). Treatment time for patient 3 was \pm 27 months (817 days).

The MARA treatment time (Table 2) for patient 1 was \pm 14 months (514 days), that for patient 2 was approximately \pm 11 months (343 days). Patient 3 had a MARA treatment time of \pm 13 months (406 days). 


\section{RESULTS}

The two main criteria that were analyzed in this study were:

- The cephalometric measurements important in diagnosis and assessment of Class II malocclusions.

- Treatment time referring to total treatment time including the initial placement and removal of full fixed appliances, and time under treatment with the MARA.

The following antero-posterior cephalometric and vertithe treatment effects of the MARA on the three cases:

\section{DISCUSSION}

\section{Treatment effects of the MARA}

The pubertal growth spurt, according to literature and previous clinical studies, ${ }^{4}$ is the most ideal time to treat Class II malocclusions resulting from mandibular deficiencies, because the growth of the jaw of the patient

The MARA produces several clinical changes which can be measured post operatively through clinical, radiographic and study model analysis.

Some of the changes observed in this study included a small degree of restriction of maxillary growth, the mesial movement of mandibular molars, pre-molars and incisors and the distalization of maxillary molars and premolars - the so-called "head gear effect". The glenoid fossa and mandibular condyles may also undergo remodeling as the mandibular condyles rest in their newly advanced position on the articular eminence of the temporal bone. ${ }^{6}$ Proffit et al. state that the remodeling is facilitated by two phenomena; the reduced pressure on the condylar tissues accompanied by a change in the muscle tension acting on the condyle. ${ }^{4}$ cal relationships (Table 1) were utilized in illustrating could be utilized and manipulated. ${ }^{9}$

\section{Mandibular changes}

The results showed a significant change in the SNB, with an average increase of $3,33^{\circ}$ in this value. The second mandibular value that is of clinical importance is the cranial base to chin point relationship or facial angle ( $\mathrm{FH} / \mathrm{N}$-Pog). The cephalometric analysis revealed an average increase of $1^{\circ}$, indicating some anterior movement of the chin.

The third value indicating notable mandibular growth in the three patients evaluated is the measurement of the distance (in millimeters) between Pt-point to A-point and Pt-point to B-point respectively. Pt-point (Pterygoid fissure) is considered a stable reference point from which the measurements were taken. The before-andafter treatment values of Pt-point to B-point revealed that there was an average increase of approximately $5,33 \mathrm{~mm}$, indicating significant horizontal/forward growth of the mandible while under treatment with the MARA.

Maxillary growth expressed as the difference in the before-and-after values of Pt-point to A-point was an average of $2,33 \mathrm{~mm}$. The resultant treatment effect of the MARA is therefore interpreted as being a relative $3 \mathrm{~mm}$ mandibular advancement (Pt-point to B-point average - Pt-point to A-point average).

Finally, an average reduction of $4^{\circ}$ and $6^{\circ}$ of the Tweed angle and $\overline{1}$ to NB angle respectively, together with reductions in the linear distance of the lower incisors to $\mathrm{NB}$, is a clear indication of mandibular advancement and mandibular incisor inclination correction toward a more favourable Class I occlusion.

\begin{tabular}{|l|c|c|c|}
\hline \multicolumn{4}{|c|}{ Table 2. The total treatment times and MARA treatment times. } \\
\hline & Patient 1 & Patient 2 & Patient 3 \\
\hline $\begin{array}{l}\text { Total Treatment Time } \\
\text { (Months) }\end{array}$ & 37 & 20 & 27 \\
$\begin{array}{l}\text { MARA Treatment Time } \\
\text { (Months) }\end{array}$ & 14 & 11 & 13 \\
\hline
\end{tabular}

\begin{tabular}{|c|c|c|c|c|c|c|}
\hline & \multicolumn{2}{|c|}{ Patient 1} & \multicolumn{2}{|c|}{ Patient 2} & \multicolumn{2}{|c|}{ Patient 3} \\
\hline & Before & After & Before & After & Before & After \\
\hline SNA & 95 & 92 & 84 & 83 & 81 & 84 \\
\hline SNB & 84 & 87 & 78 & 80 & 77 & 82 \\
\hline ANB & 11 & 5 & 6 & 3 & 4 & 2 \\
\hline WITS & 8 & 2 & 6 & 4 & 5 & 2 \\
\hline Y-axis & 59 & 58 & 67 & 67 & 67 & 62 \\
\hline Facial Angle & 90 & 91 & 86 & 85 & 90 & 93 \\
\hline Tweed Angle & 104 & 99 & 106 & 100 & 104 & 103 \\
\hline$\overline{1}$ to APO & $0 \mathrm{~mm}$ & $1 \mathrm{~mm}$ & $-3 \mathrm{~mm}$ & $-2 \mathrm{~mm}$ & $2 \mathrm{~mm}$ & $0 \mathrm{~mm}$ \\
\hline 1 to NA & 8 & 14 & 3 & 22 & 24 & 30 \\
\hline$\overline{1}$ to NB & 33 & 27 & 29 & 20 & 26 & 23 \\
\hline FH: GoGn & 21 & 17 & 18 & 17 & 16 & 12 \\
\hline GoGn: SN & 26 & 21 & 26 & 22 & 26 & 20 \\
\hline \multirow{4}{*}{$\begin{array}{l}\text { Mandibular growth } \\
\text { in } \mathrm{mm} \text { from } \\
\text { Pt-point to A\&B } \\
\text { points respectively. }\end{array}$} & \multicolumn{2}{|c|}{ Pt point to A-point } & \multicolumn{2}{|c|}{ Pt point to A-point } & \multicolumn{2}{|c|}{ Pt point to A-point } \\
\hline & $72 \mathrm{~mm}$ & $75 \mathrm{~mm}$ & $72 \mathrm{~mm}$ & $75 \mathrm{~mm}$ & $68 \mathrm{~mm}$ & $69 \mathrm{~mm}$ \\
\hline & \multicolumn{2}{|c|}{ Pt point to B-point } & \multicolumn{2}{|c|}{ Pt point to B-point } & \multicolumn{2}{|c|}{ Pt point to B-point } \\
\hline & $88 \mathrm{~mm}$ & $95 \mathrm{~mm}$ & $92 \mathrm{~mm}$ & $97 \mathrm{~mm}$ & $88 \mathrm{~mm}$ & $92 \mathrm{~mm}$ \\
\hline \multirow{4}{*}{$\begin{array}{l}\text { Mandibular growth } \\
\text { in } \mathrm{mm} \text { from Sella } \\
\text { to A\&B points } \\
\text { respectively. }\end{array}$} & \multicolumn{2}{|c|}{ Sella to A-point } & \multicolumn{2}{|c|}{ Sella to A-point } & \multicolumn{2}{|c|}{ Sella to A-point } \\
\hline & 105 & 104 & 100 & 101 & 92 & 92 \\
\hline & \multicolumn{2}{|c|}{ Sella to B-point } & \multicolumn{2}{|c|}{ Sella to B-point } & \multicolumn{2}{|c|}{ Sella to B-point } \\
\hline & 119 & 132 & 120 & 132 & 112 & 118 \\
\hline
\end{tabular}


Patient 1: Before treatment.

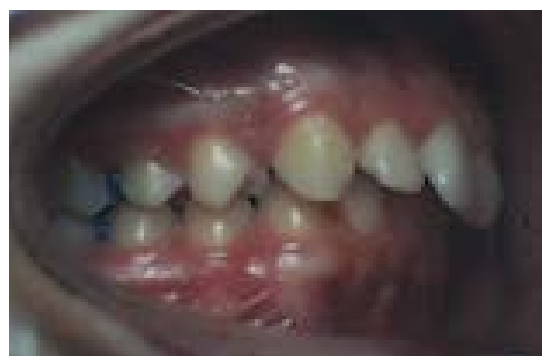

Figure 6A.

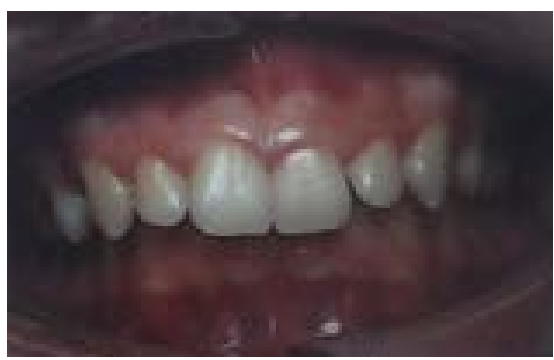

Figure 6B.

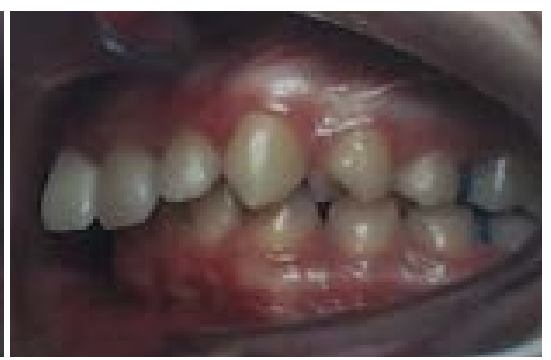

Figure 6C.

Patient 1: During treatment.

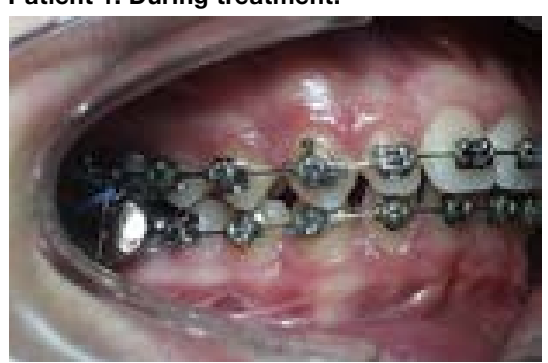

Figure 6D.

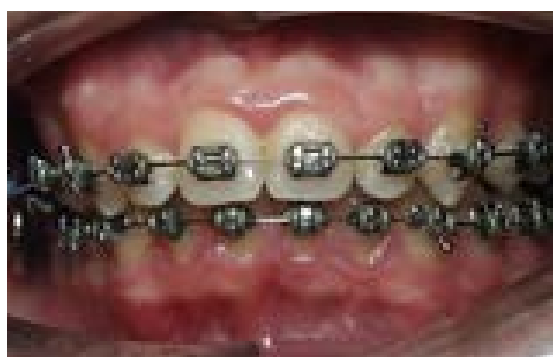

Figure 6E.

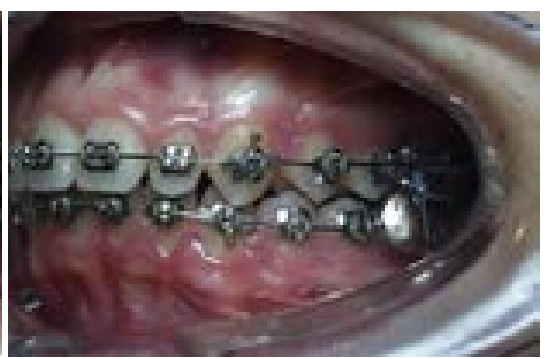

Figure 6F.

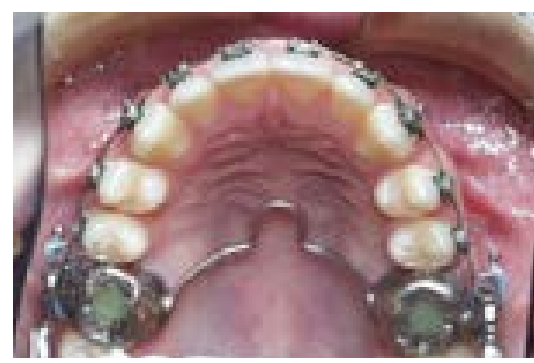

Figure 6G.

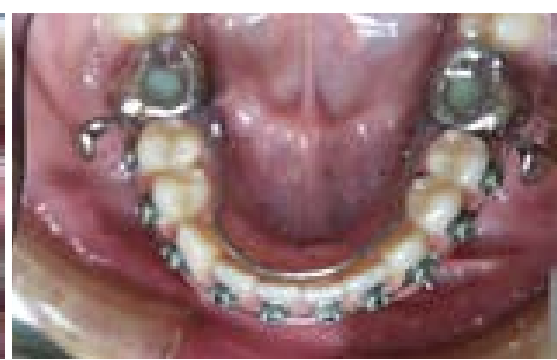

Figure 6H.

Patient 1: Treatment completed.

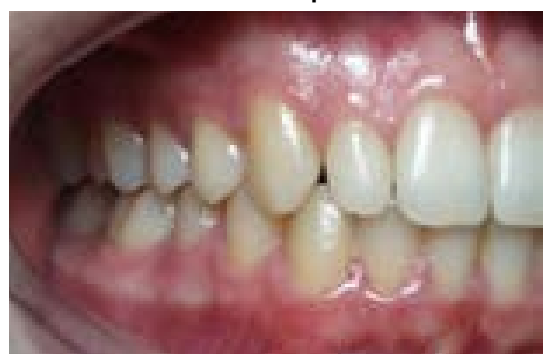

Figure 61.

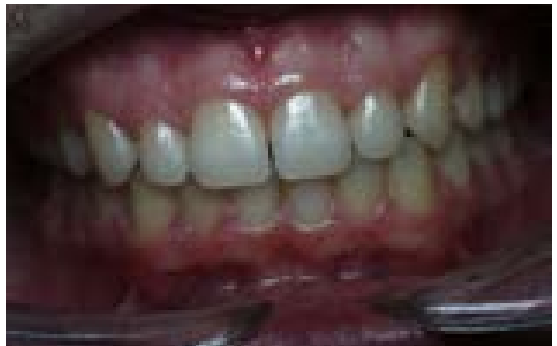

Figure 6J.

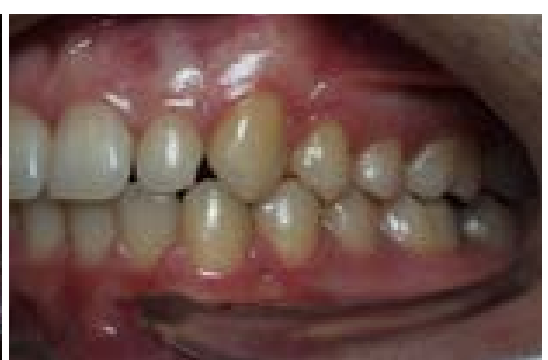

Figure 6K.

Patient 1: Facial profile before and after treatment:

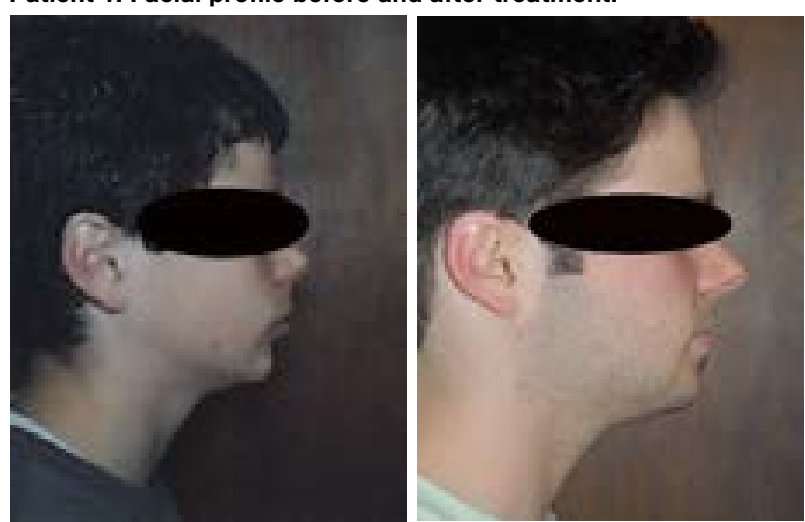

Figure 6L.

Figure 6M. Note the improvement in the nasio-labial angle. 
Appliance-induced mandibular advancement resulting in possible mandibular remodeling with subsequent anteriorly directed growth produces an improvement of the Class II malocclusion. Al-Jewair, Preston, Moll \& Dischinger ${ }^{10}$ found comparable results.

\section{Maxillary changes}

In this study, a change was also noted in the SNA value, with an average reduction of $0,33^{\circ}$ which is in accordance with the observations made during other investigations. These results indicate some maxillary growth restriction through the headgear effect produced by the MARA.

Notable intrusion of the first maxillary molars was observed on removal of the MARA. Occlusal and masticatory forces acting on the stainless steel crowns that were placed on these teeth to facilitate anchorage of the device, are responsible for this intrusion. The amount of first molar intrusion also depends on how much the bite is opened after the cementation of the stainless steel crowns. Apparent extrusion of the maxillary second molars was also noted, most probably due to the tip back on the upper first and second molars.

\section{Maxillary-Mandibular changes}

The average change obtained in the ANB angle was a reduction of $3,66^{\circ}$, resulting from mandibular anterior repositioning in combination with maxillary growth restriction, producing improvement of the Class II malocclusion.

An average reduction of $3,66 \mathrm{~mm}$ in the WITS value was observed after treatment with the MARA. The reduction in the WITS value further indicates a significant improvement of the Class II malocclusion in the treated patients.

The correction of the Class II malocclusions was achieved by several factors of which mandibular growth is the most significant. Other factors include the distalization and intrusion of the maxillary first and second molars together with the mesial movement of the mandibular molars and labial tipping of the lower incisors as well as the headgear effect on the maxilla as a whole.

The changes observed were mostly in the anteroposterior dimension with the vertical alterations being less significant.

\section{Growth direction}

Although the $\mathrm{Y}$-axis relationship is viewed with reservation (due to $S$ and $S N$ variability), it was found that the pre-treatment values were relatively normal.

Post-treatment values however showed that there was an average decrease of $2^{\circ}$ of this value, indicating a slightly more horizontal pattern of growth which is consistent with mandibular advancement and closing rotation of the chin, contributing to the resolution of the Class II malocclusions.

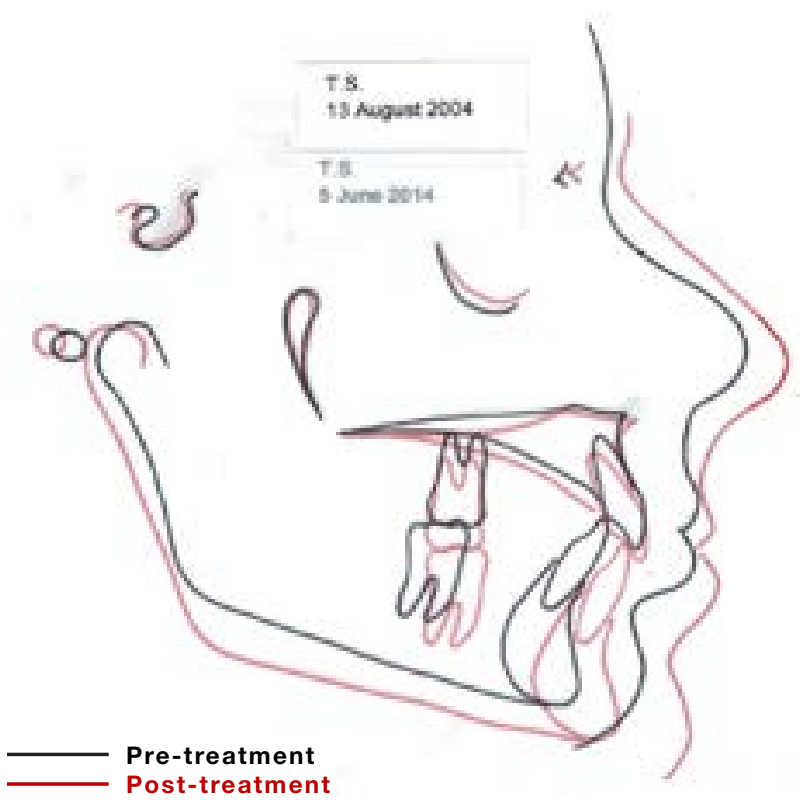

Figure 7A. Superimposition of cephalometric radiographs at Pterygoid fissure $(\mathrm{Pt})$ showing the pre-treatment situation and the total post retention status (10 years later) obtained in case 1 .

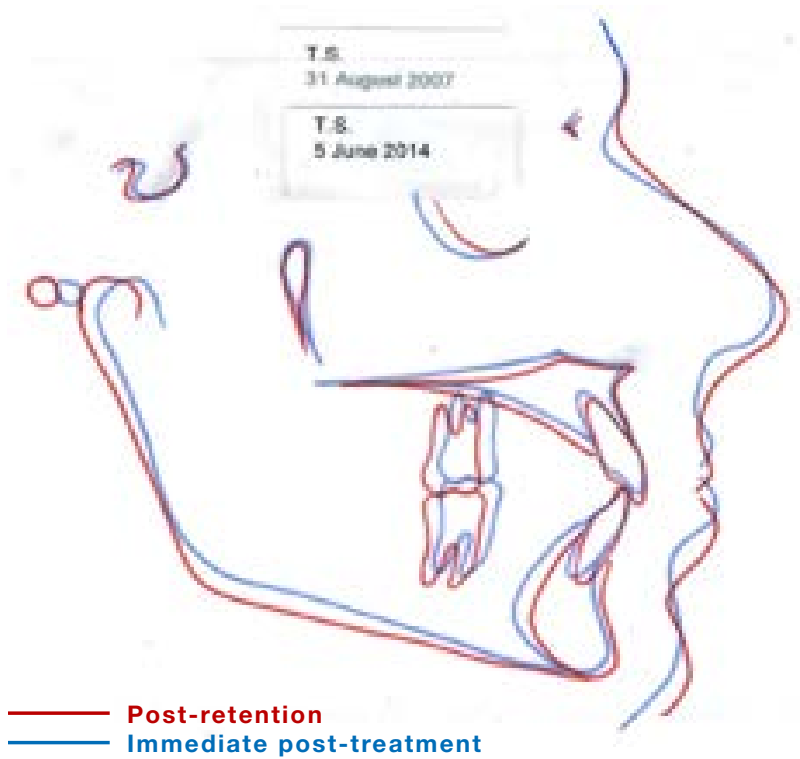

Figure 7B. Superimposition of cephalometric radiographs at $\mathrm{Pt}$-point showing long-term treatment stability obtained in case $1 \quad( \pm 7$ years following post retention). No relapse to a Class II malocclusion had occurred following MARA treatment.

\section{Treatment times}

The average total treatment time of the three cases was 28 months, while the average MARA treatment time was 12,7 months. The prolonged treatment times as seen with the first of the above three cases can be ascribed to the relative inexperience of the operator with the handling of the MARA, a conservative amount of appliance activation leading to longer treatment times and patients not necessarily complying with appointment times/schedules.

The accepted time span for the treatment of a Class II malocclusion with a fixed functional appliance like the MARA may vary from each individual case but usually ranges from $6-8$ months. $^{4}$ 
Patient 2: Before treatment.

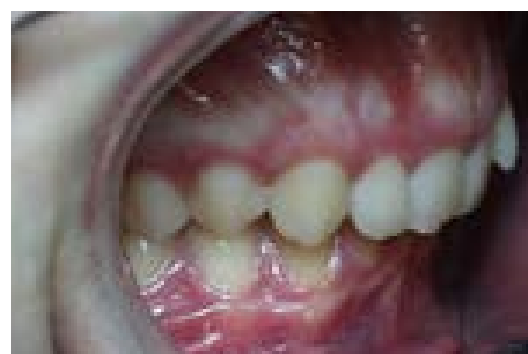

Figure 8A.

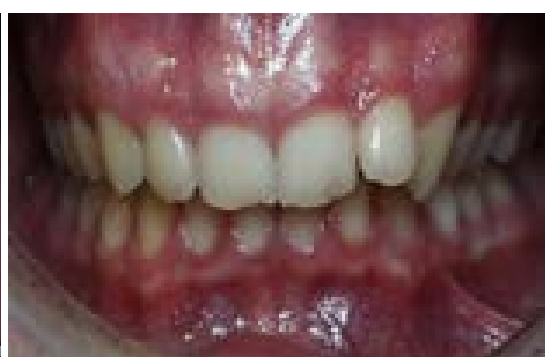

Figure 8B.

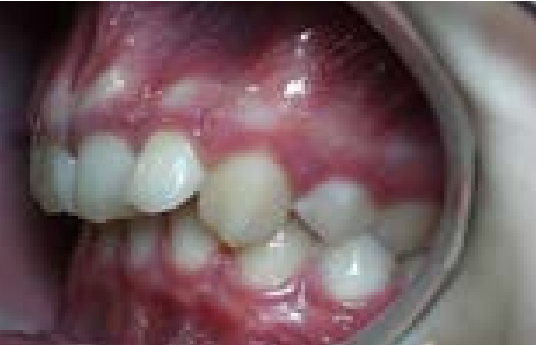

Figure 8C.

Patient 2: During treatment.

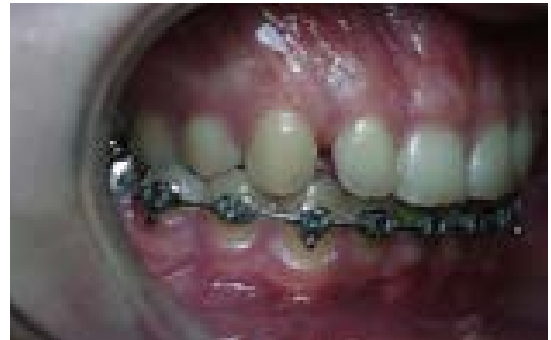

Figure 8D.

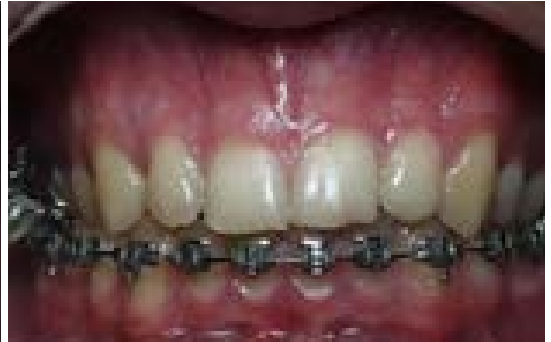

Figure 8E.

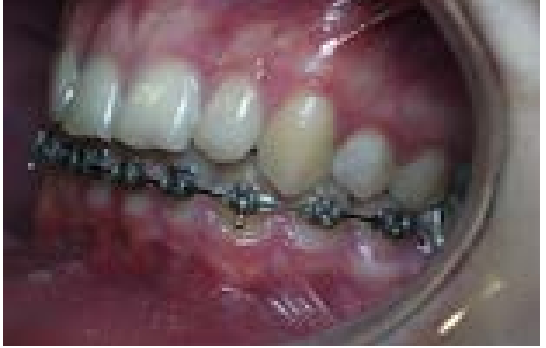

Figure 8F.

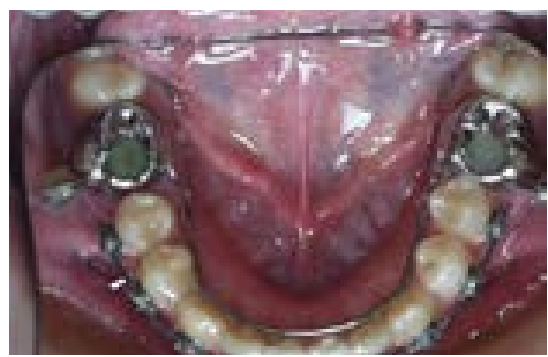

Figure 8G.

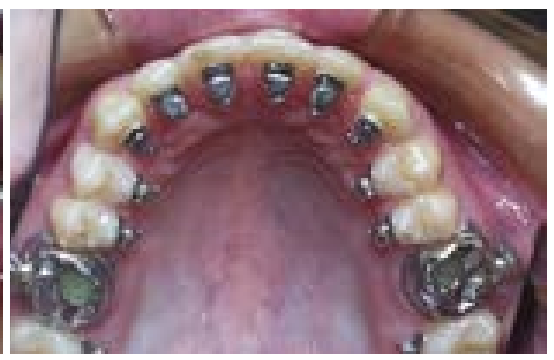

Figure $\mathbf{8 H}$.

Patient 2: Treatment completed.

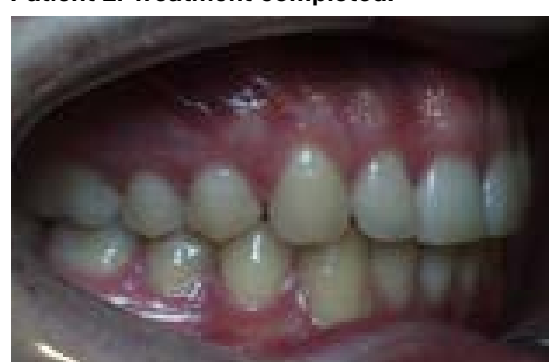

Figure 81 .

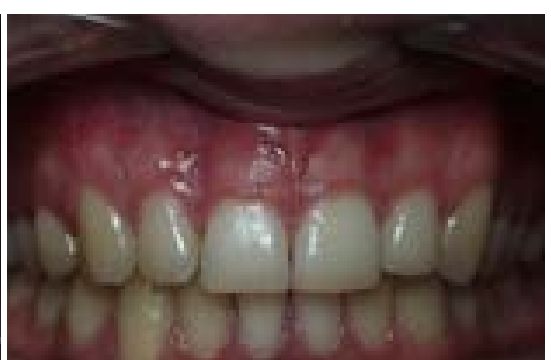

Figure 8J.

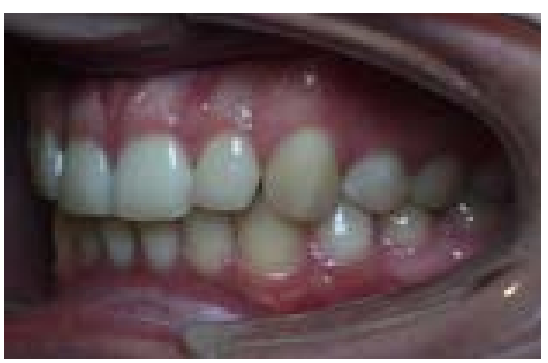

Figure 8K.

Patient 2: Facial profile before and after treatment:

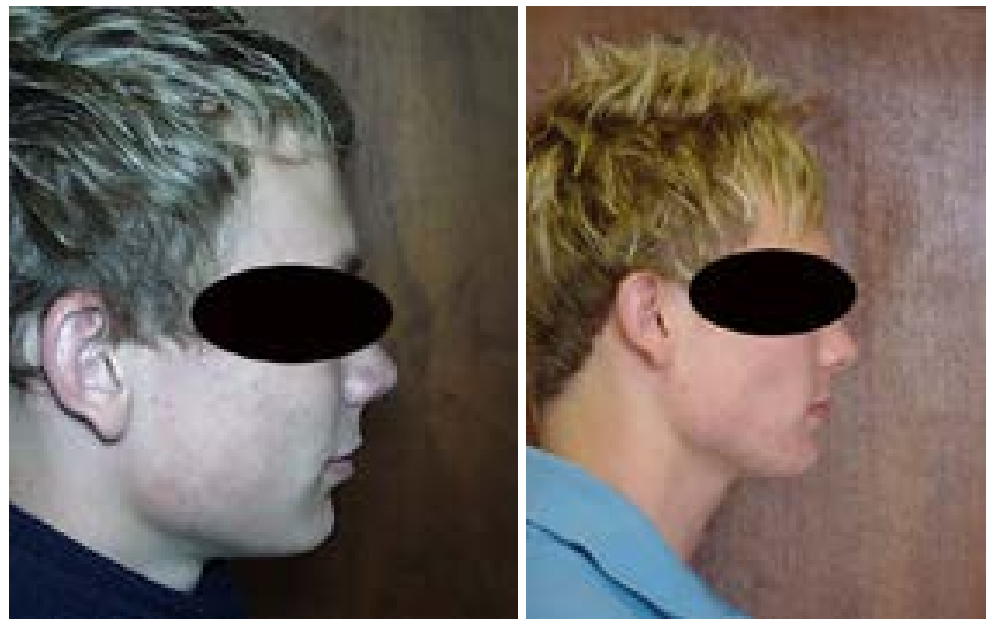

Figure 8L.

Figure 8M. 
Below is a compilation of intra-oral and facial profile images taken prior to, during and after treatment showing the MARA in combination with full fixed appliances. Correction or improvement of the Class II defect is clearly visible in all three cases (Patient 1: Figure 6a-m, Patient 2: Figure 8a-k \& Patient 3: Figure $10 \mathrm{a}-\mathrm{j})$.

Superimposition of three cephalometric radiographs with reference point at the pterygoid fissure (Pt point) was done. The treatment stages include pre-treatment, directly following treatment and several years after completion of orthodontic treatment.

The images show significant clinical and skeletal improvement of the Class II malocclusions, with the second superimposition demonstrates the post treatment stages to long-term stability of the changes obtained with the MARA (Figures 7a\&b, 9a\&b and 11a\&b).

The black tracing corresponds with the pre-treatment findings, the blue tracing demonstrates the results immediately following treatment and the red tracing a few years after treatment was completed.

\section{CONCLUSION}

The MARA is an effective fixed non-compliance appliance that works well in conjunction with full fixed appliances in the treatment of Class II malocclusions as demonstrated in the three cases reported in this study Treatment time is predictable as seen in the three cases.

The time required during the placement and removal of the MARA is more than compensated by the shorter and fewer follow up visits involved. No or very little patient motivation is necessary.

In the three reviewed cases a favourable facial profile was achieved with the nasolabial angle more desirable than that which would have been obtained with selective extractions and extra-oral traction.

The three subjects presented here may be regarded as uncomplicated cases. Experience over many years in the treatment of several MARA cases with the MARA has shown that more difficult cases can indeed be treated; often with unexpectedly favourable results.

In many of these instances the general consensus would probably have been that orthognathic surgery would be the treatment of choice.

Although orthognathic surgery is integral to the treatment of severe Class II malocclusions, the MARA may be seen as a possible alternative to certain surgical interventions, being especially useful in cases where the patient refuses surgery as a treatment option.

There will of course always be indications and contraindications for using the MARA and no claims may be made that treatment with the appliance will routinely eliminate the need for surgery in a major percentage of those patients who would otherwise be treated with surgery.

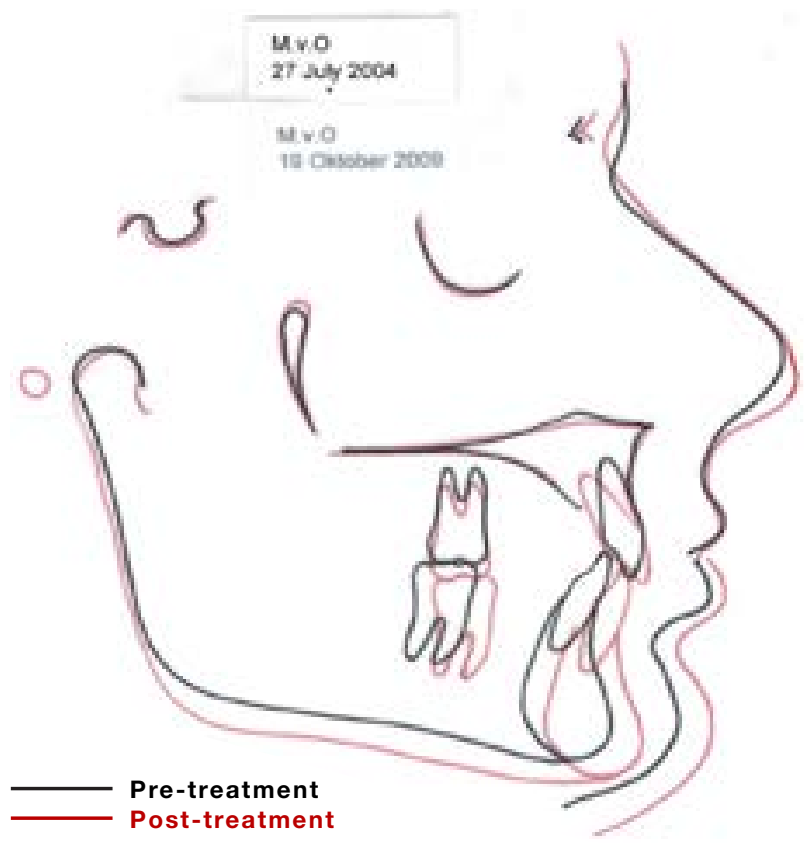

Figure 9A. Superimposition of cephalometric radiographs at Pt-showing the pre-treatment situation and the total post retention changes (5 years later) obtained in case 2.

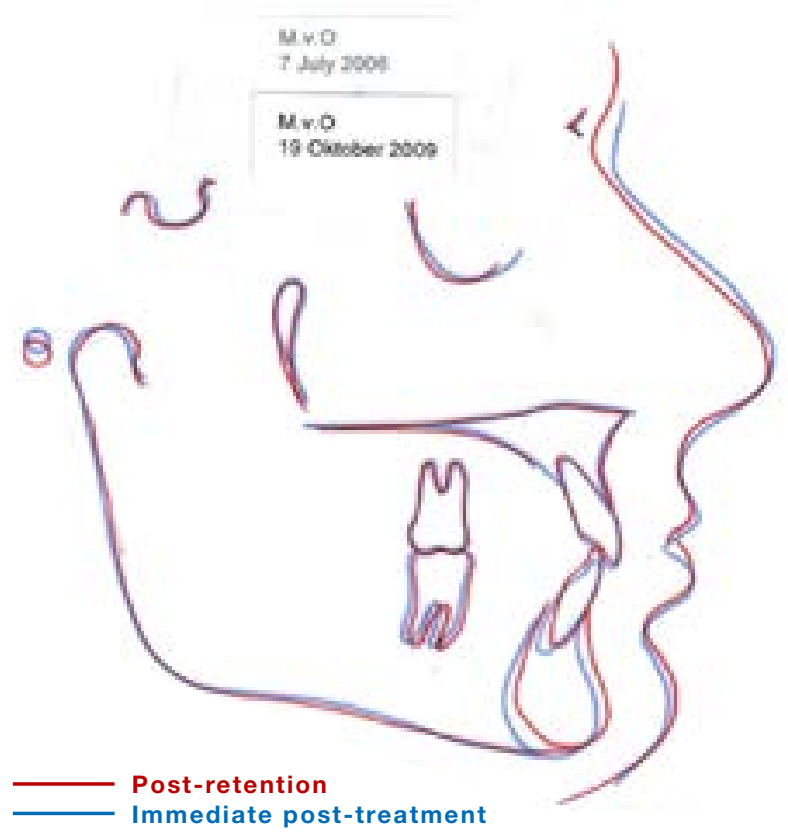

Figure 9B. Superimposition of cephalometric radiographs at Pt-point showing long-term treatment stability obtained in case 2 ( \pm 3 years following post retention). No relapse to a Class II malocclusion occurred following MARA treatment.

As always, proper diagnosis, sound judgment as well as full informed consent are important and the clinician should be careful not to create the impression that all surgery could or should be avoided.

\section{References}

1. Ghislanzoni LTH, Toll DE, Defraia E, Bacetti T, Franchi L. Treatment and post-treatment outcomes induced by the Mandibular Advancement Repositioning Appliance. Angle Orthod. 2011; 81(4): 684-91.

2. White LW, Eckhart JE. Functional non-extraction treatment. Clinical Impressions. 2009; 17(1): 32-43. 
Patient 3: Before treatment.

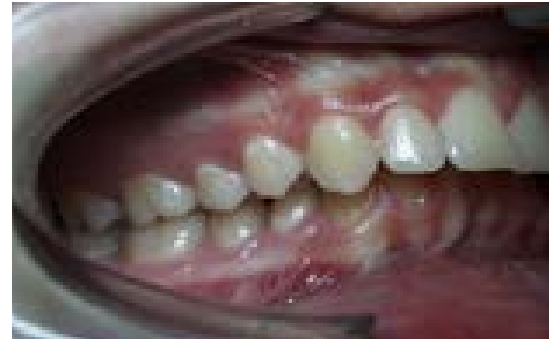

Figure 10A.

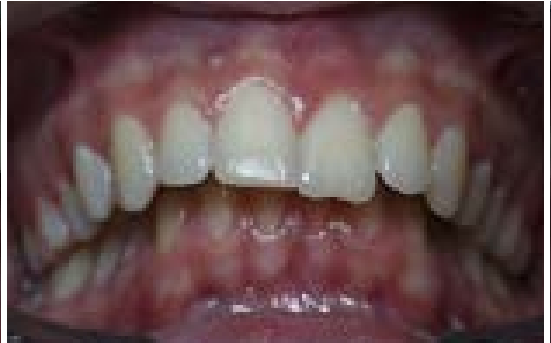

Figure 10B.

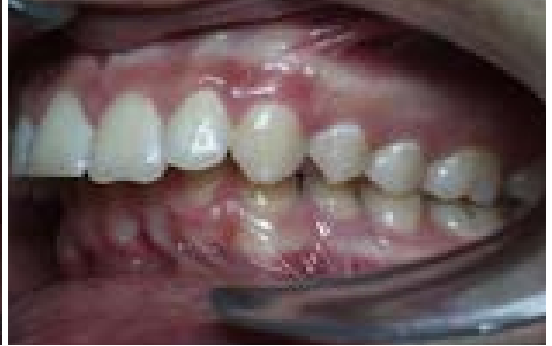

Figure 10C.

Patient 3: During treatment.

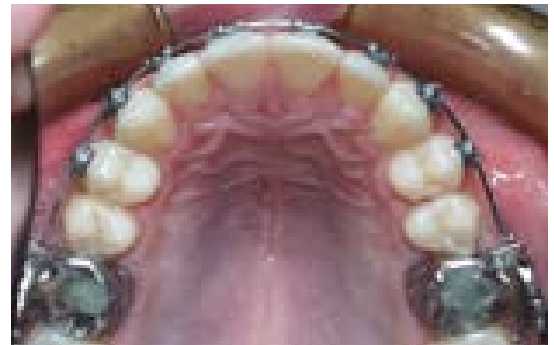

Figure 10D.

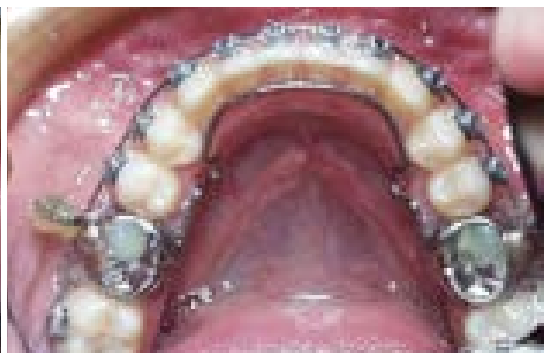

Figure 10E.

Patient 3: Treatment completed.

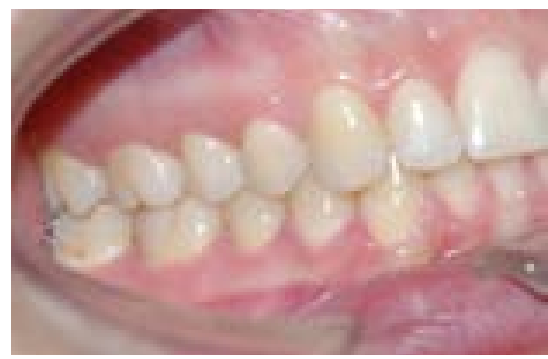

Figure 10F.

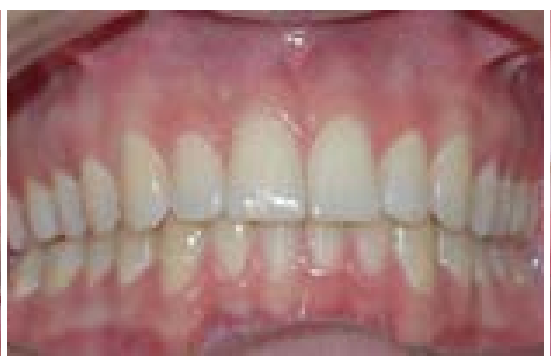

Figure 10G.

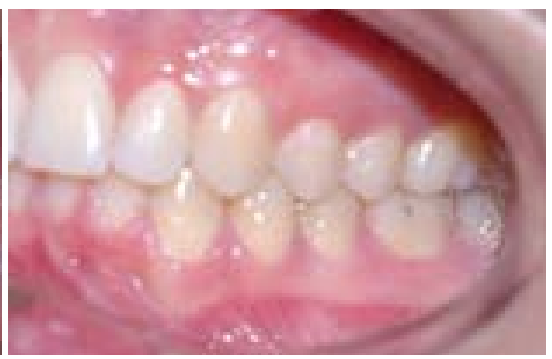

Figure 10H.

Patient 3: Facial profile before and after treatment:

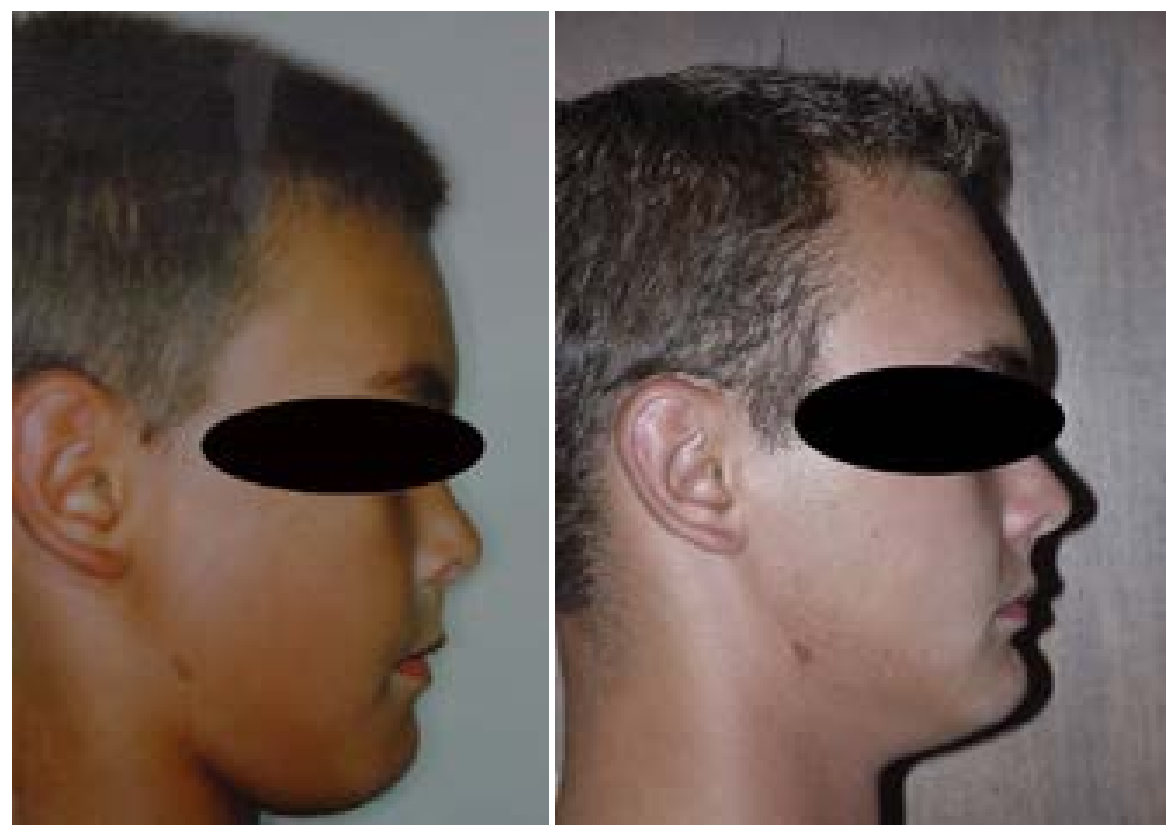




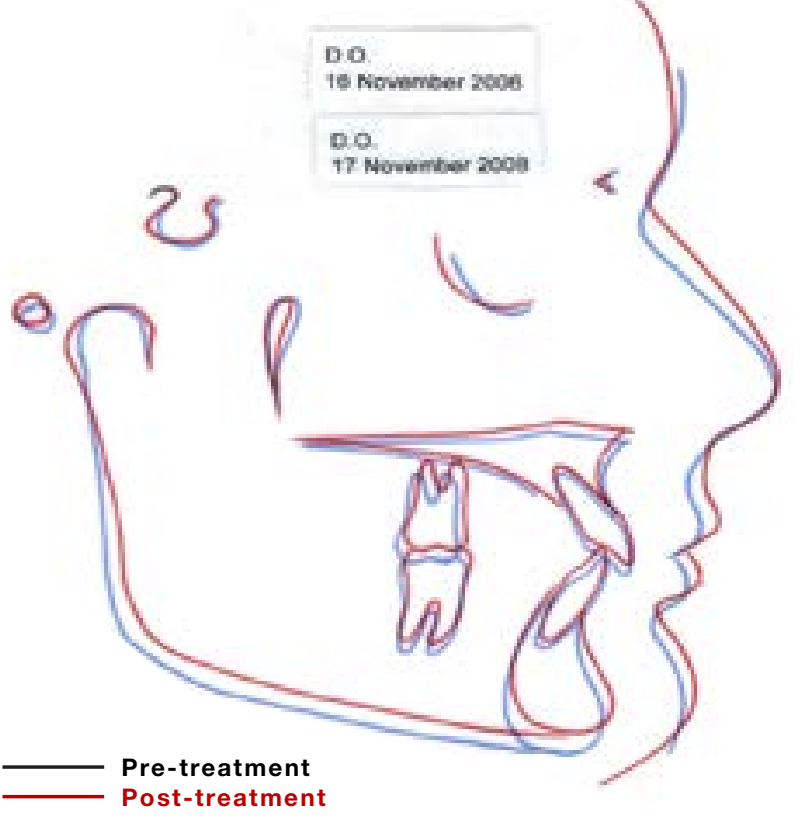

Figure 11A. Superimposition of cephalometric radiographs at Pt-showing the pre-treatment situation and the total post retention changes (5 years later) obtained in case 3 .

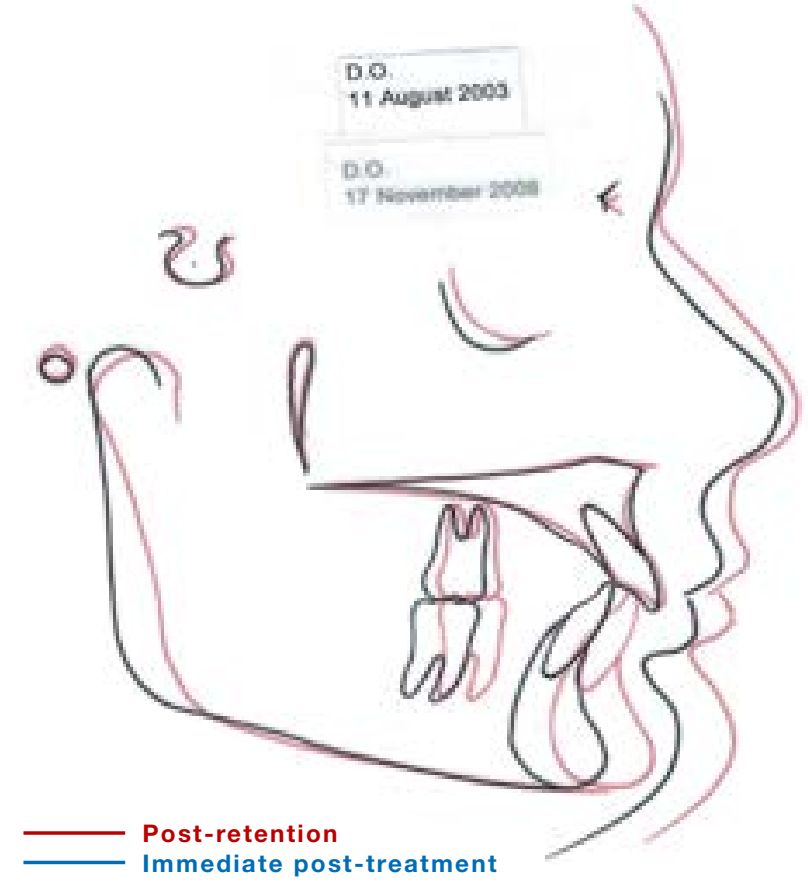

Figure 11B. Superimposition of cephalometric radiographs at Pt-point showing long-term treatment stability obtained in case 3 ( \pm 2 years following post retention). No relapse to a Class II malocclusion occurred following MARA treatment.
3. Pangrazio MNK, Pangrazio-Kulberch V, Berger JL, Bayirli B, Movahhedian A. Treatment effects of the mandibular anterior repositioning appliance in patients with Class II skeletal malocclusions. Angle Orthod. 2012; 82(6): 971-7.

4. Proffit WR, Fields HW. Contemporary Orthodontics, $3^{\text {th }}$ ed. St. Louis MI. Mosby, 2000.

5. Keim RG, Gottlieb EL, Nelson AH, Vogels DS. Study of orthodontic diagnosis and treatment procedures, Part 1: Results and Trends. J Clin Orthod. 2008; 42(11): 625-40.

6. Eckhart JE. Introducing the MARA. Clinical Impressions. 1998; 7(3): 2-5.

7. Bogdan F. The MARA: A noncompliance solution. Clinical Impressions. 2006; 15(1): 28-32.

8. Eckhart JE. MARA provides effective adult treatment. Clinical Impressions. 2001; 10(1): 16-8.

9. Ghislanzoni LTH, Baccetti T, Toll D, Defraia E, McNamara JA, Franchi $L$. Treatment timing of MARA and fixed appliance therapy of Class II malocclusion. Eur J Orthod. 2013; 35: 394-400.

10. Al-Jewair ST, Preston CB, Moll E, Dischinger T. A comparison of the MARA and the AdvanSyncfunctional appliances in the treatment of Class II malocclusion. Angle Orthod. 2012; 82(5): 907-14. 